\title{
Probabilistic Models and Generative Neural Networks: Towards an Unified Framework for Modeling Normal and Impaired Neurocognitive Functions
}

\author{
Alberto Testolin ${ }^{1 *}$ and Marco Zorzi ${ }^{1,2 *}$ \\ ${ }^{1}$ Department of General Psychology and Center for Cognitive Neuroscience, University of Padova, Padua, Italy, ${ }^{2}$ IRCCS San \\ Camillo Neurorehabilitation Hospital, Venice-Lido, Italy
}

Connectionist models can be characterized within the more general framework of probabilistic graphical models, which allow to efficiently describe complex statistical distributions involving a large number of interacting variables. This integration allows building more realistic computational models of cognitive functions, which more faithfully reflect the underlying neural mechanisms at the same time providing a useful bridge to higher-level descriptions in terms of Bayesian computations. Here we discuss a powerful class of graphical models that can be implemented as stochastic, generative neural networks. These models overcome many limitations associated with classic connectionist models, for example by exploiting unsupervised learning

OPEN ACCESS

Edited by:

Eirini Mavritsaki,

Birmingham City University, UK

Reviewed by:

Marcel Van Gerven,

Radboud University Nijmegen,

Netherlands

Julien Mayor,

The University of Nottingham

Malaysia Campus, Malaysia

*Correspondence:

Alberto Testolin alberto.testolin@unipd.it

Marco Zorzi

marco.zorzi@unipd.it

Received: 27 May 2016 Accepted: 30 June 2016 Published: 13 July 2016

Citation:

Testolin A and Zorzi M (2016) Probabilistic Models and Generative Neural Networks: Towards an Unified Framework for Modeling Normal and Impaired Neurocognitive Functions. Front. Comput. Neurosci. 10:73. doi: $10.3389 /$ fncom.2016.00073 in hierarchical architectures (deep networks) and by taking into account top-down, predictive processing supported by feedback loops. We review some recent cognitive models based on generative networks, and we point out promising research directions to investigate neuropsychological disorders within this approach. Though further efforts are required in order to fill the gap between structured Bayesian models and more realistic, biophysical models of neuronal dynamics, we argue that generative neural networks have the potential to bridge these levels of analysis, thereby improving our understanding of the neural bases of cognition and of pathologies caused by brain damage.

Keywords: connectionist modeling, unsupervised learning, deep neural networks, probabilistic generative models, computational neuropsychology

\section{INTRODUCTION}

Despite the enormous progress in the prevention and treatment of neuropsychological disorders, traumatic brain injury and stroke are still among the major causes of adult disability and death (Mathers et al., 2008; Feigin et al., 2014). This social impact highlights the importance of neuropsychological research and the recent thrust in supporting empirical investigations with modern computational tools (Gerstner et al., 2012). In particular, network-based models of brain function conceive cognitive processes as complex phenomena emerging from the simultaneous interaction of many constituent components, and are therefore particularly suited to study the effects of brain damage from a computational perspective (O’Reilly and Munakata, 2000).

One of the most successful attempts to ground neuropsychology within a computational framework has been achieved by parallel distributed processing (PDP) models 
(Rumelhart and McClelland, 1986), which describe cognition as the evolution over time of a system of interconnected units that self-organize according to physical principles. Within this framework, the pattern seen in overt behavior (macroscopic dynamics of the system) reflects the operations of subcognitive processes (microscopic dynamics of the system), such as the propagation of activation and inhibition among simple processing units. A distinguishing feature of PDP models is their ability to adapt to the environment, which allows to simulate behavioral patterns associated with a broad range of cognitive functions and to study how learning mechanisms support cognitive development and knowledge acquisition (e.g., Elman et al., 1996). Crucially, the tight link between structure and function in PDP models allows to investigate how changes in the underlying processing mechanisms are reflected by changes in overt behavior, thereby providing a principled way to simulate neuropsychological disorders following brain damage (e.g., Hinton and Shallice, 1991; Plaut and Shallice, 1993; McClelland et al., 1995).

However, despite the broad range of cognitive functions (and cognitive disorders) investigated through this approach, many PDP models suffer from serious limitations. In particular, connectionist models are often trained in a supervised fashion using error backpropagation, but the assumption that learning is largely discriminative and that an external teaching signal is available at each learning event is implausible from a cognitive perspective (see Zorzi et al., 2013, for discussion). Moreover, besides the need for labeled patterns, classic PDP models usually entail an over-simplistic, "shallow" processing architecture, involving only one layer of hidden units and strictly feed-forward connectivity. This is in sharp contrast with well-known properties of cortical circuits, which exhibit a hierarchical organization (Felleman and Van Essen, 1991) where information processing relies on both feed-forward and feedback mechanisms (Sillito et al., 2006; Gilbert and Sigman, 2007). Finally, these processing constraints (together with limitations in computational power) have prevented to extend "toy models" into large-scale simulations of neural networks composed by thousands of neurons and millions of connection weights that can be trained using realistic input patterns.

The aim of this article is to describe a new generation of PDP models that address these limitations. In particular, we discuss how they have been exploited for modeling a wide range of neurocognitive functions, and we highlight their potential for simulating neuropsychological deficits.

\section{A NEW GENERATION OF PARALLEL DISTRIBUTED PROCESSING MODELS}

Probabilistic graphical models provide a general approach to model the stochastic behavior of a large number of interacting variables, whose relations are efficiently represented using graphical structures (Koller and Friedman, 2009). Notably, many PDP models can be characterized within this probabilistic framework (Jordan and Sejnowski, 2001). In particular, a powerful class of stochastic, recurrent neural networks can be characterized as fully-connected graphical models, where the undirected nature of the edges implies bidirectional flow of information between the nodes (Ackley et al., 1985). This probabilistic interpretation of neural networks provides a useful bridge to more abstract computational descriptions of cognitive processes (Griffiths et al., 2008), suggesting how high-level Bayesian computations might be implemented in neural circuits. Indeed, the problem of finding the best possible interpretation of an ambiguous stimulus can be formalized as an unconscious, statistical inference process. A possible role for recurrent feed-forward/feedback loops in the cerebral cortex might therefore be to integrate top-down, contextual priors with bottom-up, sensory observations, so as to implement concurrent probabilistic inference along the whole cortical hierarchy (Lee and Mumford, 2003; McClelland, 2013).

\section{Unsupervised Learning in Generative Neural Networks}

Learning in probabilistic graphical models can be framed within two different settings. In discriminative learning, the goal is to model only conditional distributions over a set of target variables, whose values are specified by associating an explicit label to each observed pattern. In generative learning, instead, the aim is to model the joint distribution of all the variables in the model, thus including also the observed variables. Notably, generative models can be efficiently implemented as stochastic neural networks that learn to reconstruct the sensory input (maximumlikelihood learning) through feedback connections and Hebbianlike learning mechanisms (Hinton, 2002). From a cognitive modeling perspective, these models are appealing because they can build high-level, distributed representations of the data by extracting statistical regularities in a completely unsupervised way (Zorzi et al., 2013). Moreover, feedback connections have a primary role in generative networks because they carry top-down expectations of the model, which are updated during learning in order to better reflect the observed sensory data (Hinton et al., 1995).

Simple generative networks can be used as building blocks for more complex architectures, such as those used in deep learning systems, where the hidden variables of the generative model are hierarchically organized (Hinton and Salakhutdinov, 2006). Hierarchical generative models efficiently structure the representation space by promoting features reuse: simple features extracted at lower levels can be successively combined to create more complex features, which eventually unveil the main causal factors underlying the data distribution (Hinton, 2007). Moreover, these high-level, abstract representations of the sensory data can also easily support supervised read-outs (Testolin et al., 2013; Zorzi et al., 2013; Figure 1A).

Generative networks have also been extended to the temporal domain (e.g., Sutskever et al., 2008), where input patterns appear in a precise, sequential order. In this case, statistical inference is performed by considering, besides the current observed evidence, also the history provided by the temporal context, which is propagated through delayed connections (Figure 1B). 


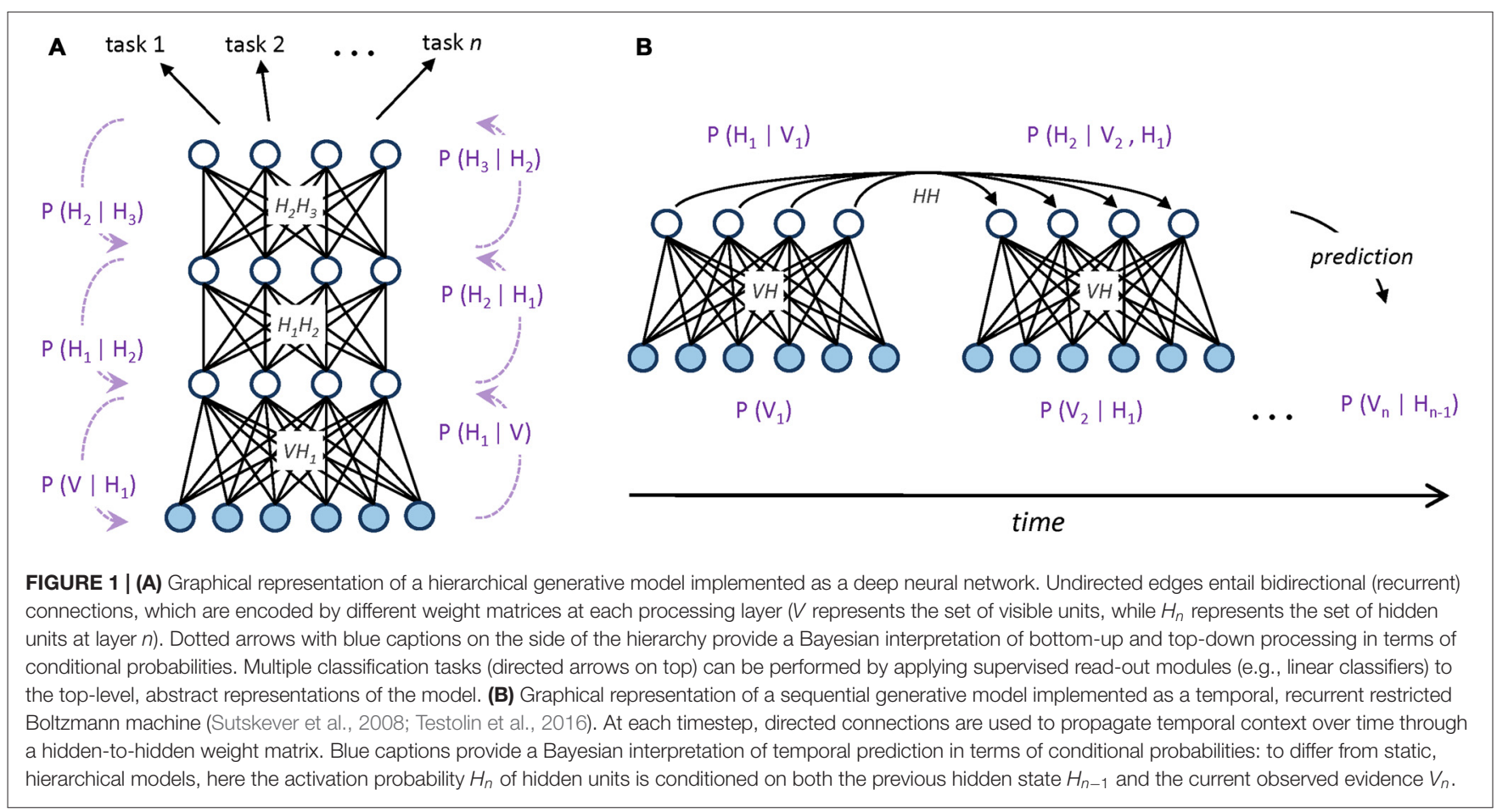

Extracting temporal dependencies is a formidable challenge for the brain (Dehaene et al., 2015), but it leads to more powerful internal models of the environment that can be used to actively predict the sensory stream (Friston, 2010; Clark, 2013). The ability to anticipate external events is also crucial for attentional mechanisms, which efficiently select sensory information according to top-down expectations and current goals (Corbetta and Shulman, 2002). In this respect, generative models allow to conceive attention as an intrinsic property of bidirectional processing networks (Casarotti et al., 2012) and to use information theoretic measures to operationalize properties like novelty/surprise in terms of discrepancy between model's expectation and observed sensory evidence (Itti and Baldi, 2009).

Finally, deep learning systems coupled with reinforcement learning algorithms have recently obtained state-of-the-art performance in extremely challenging cognitive tasks, for example by learning to play videogames at human-level (Mnih et al., 2015) or by defeating professional players on difficult board games (Silver et al., 2016). This powerful learning modality takes into account the effects of actions on the environment without requiring an explicit supervision signal, and therefore would constitute a cognitively (Botvinick et al., 2009) and biologically (Gläscher et al., 2010) plausible way to couple unsupervised deep learning with goal-directed behavior.

\section{Recent Neurocognitive Models}

In the domain of numerical cognition, unsupervised deep learning has been successfully used to show how visual numerosity could emerge as a statistical property of images containing a variable number of items (Stoianov and Zorzi, 2012; Figure 2A). Numerosity detectors developed by the network had response profiles resembling those of monkey parietal neurons (Roitman et al., 2007), and supported numerosity estimation with the same behavioral signature shown by humans and animals. A subsequent study simulated typical and atypical developmental trajectories through incremental learning and manipulation of the computational resources (i.e., number of hidden units) of the generative model (Stoianov and Zorzi, 2013), in line with the reduced gray matter density in the intraparietal sulcus observed in dyscalculic subjects (Rotzer et al., 2008). Generative networks have also been used to model learning of arithmetic facts as joint distributions of operands and results, and to simulate acquired acalculia (Stoianov et al., 2004; Zorzi et al., 2005).

Another major cognitive domain that has been modeled within this framework is that of visual object recognition, where the hierarchical representations emerging in deep networks show remarkable similarities with those recorded in the ventral visual pathway of the human brain (Güçlü and van Gerven, 2015). Unsupervised deep learning has also been recently applied to model human-like letter perception (Testolin et al., under review), where visual primitives extracted from natural scenes are later recycled for learning letters (Figure 2B) thereby supporting the hypothesis that the shape of visual symbols has been culturally selected to match the statistical structure found in our visual environment (Dehaene and Cohen, 2007). Perception of single letters can also be extended to model visual word recognition (Di Bono and Zorzi, 2013; Zorzi et al., 2013), and a temporal version of the model has been used to learn the statistical structure of letter sequences and to simulate spontaneous generation of words and pseudowords (Testolin et al., 2016). These generative networks can be used as building blocks to develop more realistic models of visual word recognition, paving the way for full-blown 
A

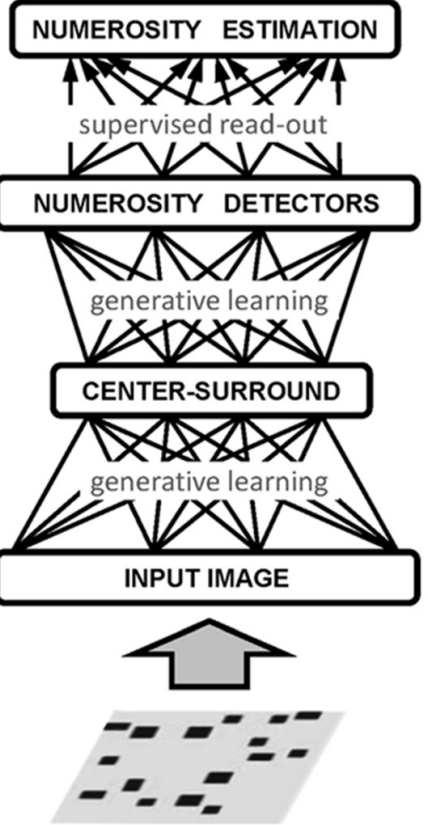

C

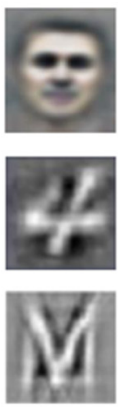

\section{c}

B OTS LETTER RECOGNITION AN supervised read-out

V2/V4

NONOS LETTER FEATURES

Мハハ ハ

generative learnin on printed letters

V1

on printed letters

VISUAL FEATURES

ハホスハ সハ

generative learning on

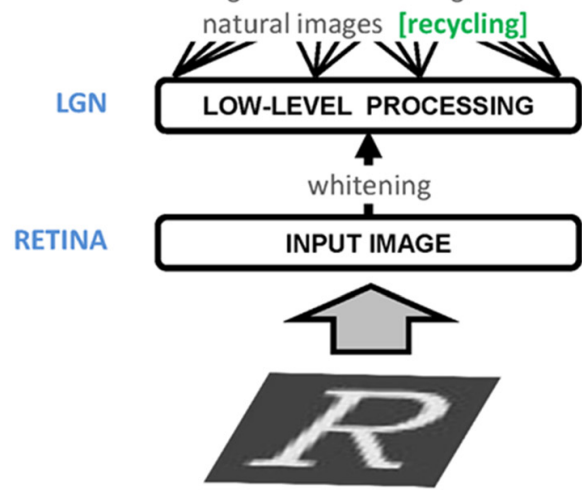

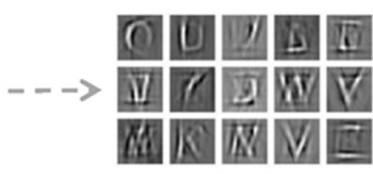
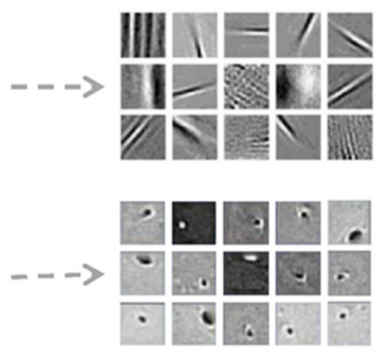

.

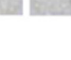

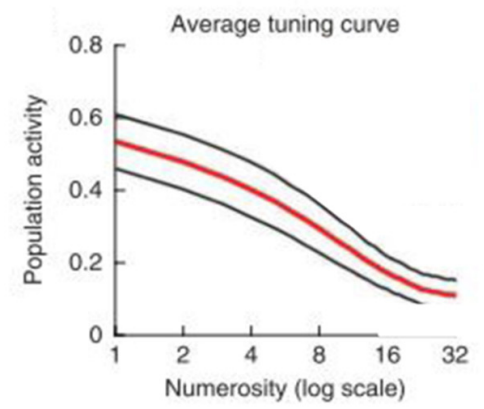

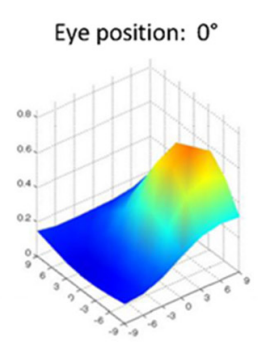

Retina coordinates

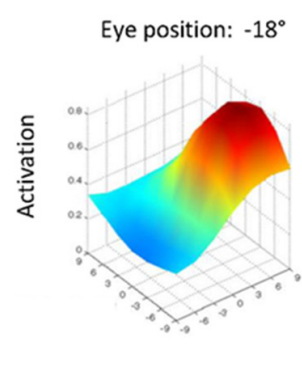

FIGURE 2 | (A) Graphical representation of the numerosity perception model of Stoianov and Zorzi (2012). A hierarchical generative model was first trained on a large set of realistic images containing visual sets with a varying number of objects. A linear read-out layer was then trained on the top-level internal representations on a numerosity comparison task. (B) Graphical representation of the letter perception model of Testolin et al. (under review). The bottom layer of the network receives the sensory signal encoded as gray-level activations of image pixels. Low-level processing occurring in the retina and thalamus is simulated using a biologically inspired whitening algorithm that captures local spatial correlations in the image and serves as a contrast-normalization step. Following generative learning on a set of patches of natural images, neurons in the first hidden layer (V1) encoded simple visual features which constitute a basic dictionary describing the statistical distribution of pixel intensities observed in natural environments. Specific learning about letters was then introduced in the model by training a second hidden layer with images containing a variety of uppercase letters. Neurons in the second hidden layer (V2N4) learned to combine V1 features to represent letter fragments and in some cases, whole letter shapes. A linear read-out layer (OTS) was then trained on the top-level internal representations in order to decode letter classes. (C) Different types of high-level features (receptive fields) emerging from unsupervised deep learning. On the left side, a prototypical face (Le et al., 2012), a prototypical handwritten digit (Zorzi et al., 2013) and a prototypical printed letter (Testolin et al., under review). In the middle panel, population activity of number-sensitive hidden neurons (mean activation value) as a function of number of objects in the display (Stoianov and Zorzi, 2012). In the right panel, a prototypical hidden neuron with a retinotopic receptive field exhibiting gain modulation (De Filippo De Grazia et al., 2012).

simulations of orthographic learning in both normal and atypical development, as well as of the impairments caused by brain damage, such as pure alexia (Plaut and Behrmann, 2011).
Generative neural networks have also been used to study space coding for sensorimotor transformations and multisensory integration (De Filippo De Grazia et al., 2012). The authors found that receptive fields reflecting those observed in 
the monkey posterior parietal cortex can emerge through unsupervised learning (Figure 2C), suggesting that gain modulation is an efficient coding strategy to integrate visual and postural information toward the generation of motor commands even though learning does not involve any explicit coordinate transformation. Notably, models of sensorimotor transformations building upon stipulated gain modulation have been used to account for visuospatial attention (Casarotti et al., 2012) and neuropsychological deficits like hemineglect (Pouget and Driver, 2000). Therefore, a promising venue for research will be to investigate these phenomena within the emergentist framework of deep generative networks.

\section{Implications for Neuropsychology}

From a neuropsychological modeling perspective, we discuss below a series of methodological advantages that this new generation of PDP models offers over more traditional connectionist models.

\section{Localized Damage Within a Hierarchical Architecture}

The structured architecture of deep learning models allows to more carefully simulate cognitive deficits caused by localized brain damage, which may affect a specific representation level. Indeed, deep networks exploit multiple levels of representation, where low-level features are gradually combined in order to produce more abstract representations of the sensory data. For example, in the domain of visual object recognition, unsupervised deep learning can lead to the emergence of extremely high-level visual features (Figure 2C), such as those representing prototypical faces (Le et al., 2012). By applying selective lesions to these models, we could assess the effect of damage to specific cortical regions, ranging from early visual processing to higher-level extrastriate areas, up to more anterior, associative areas. This would allow to simulate various forms of visual agnosia (Farah, 2004) and investigate the emergence of category-specific deficits (Humphreys and Forde, 2001). Most notably, the realistic scale of these models allows to evaluate the effect of damage using the same type of stimuli employed in patients' testing (e.g., standardized pictures of Snodgrass and Vanderwart, 1980).

\section{Multiple Connection Pathways and Multimodal Learning}

Deep learning architectures can also be used to simulate selective damage to specific connection pathways. For example, Cappelletti et al. (2014) simulated the declined performance of elderly population in numerosity comparison using the model of Stoianov and Zorzi (2012). Stochastic decay was applied to synaptic strengths to investigate two different types of impairment: a global degradation involving all network synapses, and a more selective degradation involving only the inhibitory synapses of a specific processing layer. The specific impairment of inhibition caused a large decrease of performance on stimuli in which irrelevant, continuous visual features competed with numerosity, mirroring the empirical data; conversely, the decline in performance following global impairment was identical across conditions. In line with an inhibition deficit hypothesis, the authors concluded that reduced inhibition of irrelevant information is critical to explain the specific pattern of impaired performance observed in aging. Selective damaging of connection pathways is also interesting in the context of multimodal deep learning (Ngiam et al., 2011). For example, learning a shared representation for arithmetic facts presented in both semantic and symbolic formats produces two different subnetworks that can be selectively damaged to simulate different patterns of acquired acalculia (Stoianov et al., 2004).

\section{Balance Between Bottom-Up and Top-Down Processing}

The prominent role of feedback connections in generative networks also allows to simulate unbalancing between topdown and bottom-up integration mechanisms, which are thought to underlie positive symptoms commonly observed in psychiatric disorders (Manford and Andermann, 1998). Hierarchical generative models have been used to simulate visual hallucinations in the Charles Bonnet syndrome (Reichert et al., 2013), suggesting that impaired homeostatic regulation of feedforward and feedback neuronal activity might be responsible for a wide range of symptoms observed in patients.

\section{Noise Might not Always be Detrimental}

Another major difference with respect to traditional connectionist models relates to the role of noise in simulating brain damage. Injection of noise in the activation of hidden units has been often used as a way to simulate brain damage by disrupting internal representations (e.g., Joanisse and Seidenberg, 1999). In stochastic models, instead, adding noise allows for a more efficient exploration of the network state space and helps settling into more stable attractors (Kirkpatrick et al., 1983). This is compatible with the hypothesis that neuronal noise has a key computational role in the brain, for example by keeping it in a "metastable" state that facilitates flexible settling into the most appropriate configuration (Kelso, 2012). Notably, this might also explain how structured fluctuations of brain activity, such as those observed during resting state, could emerge from noise-driven explorations of oscillatory states (Deco et al., 2013).

\section{From Toy Models to Realistic, Large-Scale Simulations}

Finally, the appeal of generative neural networks has long been hindered by their high computational complexity. This has been radically changed by recent advances in parallel computing architectures, which allow to efficiently simulate large-scale neural networks composed by thousands of neurons (Raina et al., 2009; Testolin et al., 2013) that can be trained and tested using the same type of stimuli adopted in empirical research (Stoianov and Zorzi, 2012; Güçlü and van Gerven, 2015). This increased realism will have important benefits for neuropsychological modeling, which traditionally relied on small-scale, "toy-models" that cannot reproduce realistic experimental settings. 


\section{PERSPECTIVES AND FUTURE CHALLENGES}

An important challenge will be to more closely link generative networks with structured Bayesian models (Ghahramani, 2015), which can successfully simulate a wide variety of high-level cognitive functions ranging from one-shot learning (Lake et al., 2015) to inferring causal relations, categories and hidden properties of objects, and meanings of words (see Tenenbaum et al., 2011, for discussion).

At the opposite end, bridging generative networks to more realistic neuronal models that incorporate biophysical details is another major challenge. The popularity of supervised deep learning both in academic and industry research (LeCun et al., 2015) has offset research on generative models, which nevertheless entail a more psychologically-plausible learning regimen as well as more biologically-plausible processing mechanisms (Zorzi et al., 2013; Cox and Dean, 2014). We believe, however, that generative networks will have an increasingly central role in neurocognitive modeling because they can simulate both evoked (feed-forward) and intrinsic (feedback) brain activity, where top-down mechanisms generate and maintain active representations that are modulated, rather than determined, by sensory information (Fiser et al., 2010). In this respect, although the classical approach in cognitive neuroscience has been to study neuronal responses to stimuli during task performance, the importance of intrinsic activity in shaping brain dynamics is now widely recognized (Raichle, 2015). Accordingly, spontaneous activity might not reflect trivial noisy fluctuations, because it is organized into clear spatiotemporal profiles that might reflect the functional architecture of the brain (Greicius et al., 2003; Buckner et al., 2008). The fact that intrinsic activity persists during sleep suggests its potential role in development and plasticity (Raichle, 2015), which is in line with previous attempts to characterize learning in generative networks as being driven by "wake" and "sleep" phases (Hinton et al., 1995). Nevertheless, resting activity is likely supported by dynamics emerging from synchronous oscillations of different brain areas over multiple frequency bands (Engel et al., 2001; Varela et al., 2001), but PDP models usually adopt processing units that are characterized by a single, real value representing the average activity of a neural ensemble. This implies that potentially important phase relations between spikes are completely lost. A possible way to address this limitation could be to integrate generative networks with spiking models, which can also perform near-optimal Bayesian inference (Rao, 2004; Ma et al., 2006; Deneve, 2008) or implement efficient belief propagation schemes in generic graphical models (Pecevski et al., 2011). Alternatively, networks of spiking neurons can perform probabilistic inference, thereby emulating Boltzmann machines, using an efficient but biologically realistic sampling scheme that explains many functional aspects of low-level brain dynamics, such as refractory mechanisms and finite durations of postsynaptic potentials (Buesing et al., 2011). Moreover, related models have shown how maximum-likelihood learning might occur in this type of networks by exploiting spike-timing dependent plasticity, which could be facilitated by

\section{BOX 1 | OUTSTANDING QUESTIONS}

- Current deep learning research is mostly focused on supervised learning and feed-forward convolutional networks trained with error backpropagation (LeCun et al., 2015), which have also been used to model cortical processing (e.g., Khaligh-Razavi and Kriegeskorte, 2014). How well do generative/recurrent vs. discriminative/feed-forward models compare with respect to simulating neurophysiological data and the effect of network damage?

- Feature detectors emerging in deep networks can be extremely complex and specialized. How does this relate to the theoretical debate on localist vs. distributed representations (e.g., Bowers, 2009)? Is it possible to learn a form of explicit, localistic coding that retains the advantages provided by distributed representations? What is the theoretical implication for computational modeling in neuropsychology?

- Is it possible to simulate the emergence of brain-like structural properties, such as small-worldness and rich-club organization, by starting from a general deep learning architecture? Do we need to include additional constraints (e.g., topological, metabolic)? How do learning regularizers (e.g., sparsity, weight decay, drop-out) compare with respect to organizational principles of biological neuronal networks?

- Can we improve lesioning studies in PDP models by taking into account structural and functional properties of the network? Could deep learning systems exhibit the same universal resilience patterns observed in other types of complex networks (Gao et al., 2016)?

other physiological mechanisms such as background oscillations and synchronous activity (Nessler et al., 2013). Notably, there have been other attempts to integrate models of spiking neurons with coarser mean-field models and neural masses, with the aim of providing multi-scale dynamical models of large-scale brain networks (Deco et al., 2008; Mavritsaki et al., 2011). Although these models are less easily interpretable in terms of high-level Bayesian learning and computation, they provide a more direct link to the vast amount of empirical data provided by modern neuroscience methods (e.g., Jirsa et al., 2010).

Finally, a largely unexplored research frontier would be to study PDP models using the powerful analytical techniques developed by network science (Albert and Barabasi, 2002; Newman, 2010), which are rapidly becoming a standard tool in neuroscience research (e.g., Bullmore and Sporns, 2009; Bressler and Menon, 2010; Medaglia et al., 2015). This would allow to more precisely characterize the relationship between structure and function in complex, self-organizing networks: indeed, in PDP models the initial processing architecture is fairly generic (e.g., for the restricted Boltzmann machine, a fully-connected bipartite graph with uniform random connections), and complex structural patterns gradually emerge as a product of learning. To the best of our knowledge, it is still unknown whether the emergent structure exhibits organizational principles that match those observed in brain networks, such as smallworldness and partial segregation into motifs (Park and Friston, 2013). Notably, it has also been shown that a resilience index of complex networks can in fact be measured using a universal resilience function, thereby unveiling the network characteristics that can enhance or diminish its robustness to damage and external perturbations (Gao et al., 2016). This surprising discovery could have a profound impact on neuropsychology, because it might allow to better understand 
how to improve fault-tolerance in neuronal networks, and how to more effectively recover network functions after damage.

In conclusion, we believe that stochastic, generative neural networks provide a unique interface between high-level descriptions of cognitive functions in terms of structured Bayesian computations and low-level, mechanistic explanations based on dynamical systems theory and simulations of networks whose connectivity and processing mechanisms can be constrained by neurobiological evidence. Such an integrated framework would allow building computational models spanning many levels of detail, capable of predicting salient aspects of behavior at varying levels of resolution at the same time guaranteeing interpretability according to different levels of abstractions (Gerstner et al., 2012). If this ambitious enterprise will succeed (see Box 1 for a list of outstanding research questions) we would have the most valuable tools to understand

\section{REFERENCES}

Ackley, D., Hinton, G. E., and Sejnowski, T. J. (1985). A learning algorithm for Boltzmann machines. Cogn. Sci. 9, 147-169. doi: 10.1207/s15516709cog 0901_7

Albert, R., and Barabasi, A. L. (2002). Statistical mechanics of complex networks. Rev. Mod. Phys. 74, 47-97. doi: 10.1103/RevModPhys.74.47

Botvinick, M. M., Niv, Y., and Barto, A. C. (2009). Hierarchically organized behavior and its neural foundations: a reinforcement learning perspective. Cognition 113, 262-280. doi: 10.1016/j.cognition.2008.08.011

Bowers, J. S. (2009). On the biological plausibility of grandmother cells: implications for neural network theories in psychology and neuroscience. Psychol. Rev. 116, 220-251. doi: 10.1037/a0014462

Bressler, S. L., and Menon, V. (2010). Large-scale brain networks in cognition: emerging methods and principles. Trends Cogn. Sci. 14, 277-290. doi: 10.1016/j. tics.2010.04.004

Buckner, R. L., Andrews-Hanna, J. R., and Schacter, D. L. (2008). The brain's default network: anatomy, function and relevance to disease. Ann. N Y Acad. Sci. 1124, 1-38. doi: 10.1196/annals.1440.011

Buesing, L., Bill, J., Nessler, B., and Maass, W. (2011). Neural dynamics as sampling: a model for stochastic computation in recurrent networks of spiking neurons. PLoS Comput. Biol. 7:e1002211. doi: 10.1371/journal.pcbi.10 02211

Bullmore, E., and Sporns, O. (2009). Complex brain networks: graph theoretical analysis of structural and functional systems. Nat. Rev. Neurosci. 10, 186-198. doi: $10.1038 / \mathrm{nrn} 2575$

Cappelletti, M., Didino, D., Stoianov, I., and Zorzi, M. (2014). Number skills are maintained in healthy ageing. Cogn. Psychol. 69, 25-45. doi: 10.1016/j. cogpsych.2013.11.004

Casarotti, M., Lisi, M., Umiltà, C., and Zorzi, M. (2012). Paying attention through eye movements: a computational investigation of the premotor theory of spatial attention. J. Cogn. Neurosci. 24, 1519-1531. doi: 10.1162/jocn_a_00231

Clark, A. (2013). Whatever next? Predictive brains, situated agents and the future of cognitive science. Behav. Brain Sci. 36, 181-204. doi: 10. 1017/S0140525X12000477

Corbetta, M., and Shulman, G. L. (2002). Control of goal-directed and stimulusdriven attention in the brain. Nat. Rev. Neurosci. 3, 201-215. doi: 10. 1038/nrn755

Cox, D. D., and Dean, T. (2014). Neural networks and neuroscienceinspired computer vision. Curr. Biol. 24, R921-R929. doi: 10.1016/j.cub.2014. 08.026

De Filippo De Grazia, M., Cutini, S., Lisi, M., and Zorzi, M. (2012). Space coding for sensorimotor transformations can emerge through unsupervised learning. Cogn. Process. 13, S141-S146. doi: 10.1007/s10339-012-0478-4 how neuronal processes support complex behavior and cognition, how brain damage impairs performance, and how to devise intervention strategies to improve recovery of function.

\section{AUTHOR CONTRIBUTIONS}

AT and MZ equally contributed to the conception and writing of the manuscript. AT and MZ are accountable for all aspects of the work in ensuring that questions related to the accuracy or integrity of any part of the work are appropriately investigated and resolved.

\section{ACKNOWLEDGMENTS}

This research was supported by grants from the European Research Council (No. 210922) and by the University of Padova (Strategic Grant NEURAT) to MZ.

Deco, G., Jirsa, V. K., and McIntosh, A. R. (2013). Resting brains never rest: computational insights into potential cognitive architectures. Trends Neurosci. 36, 268-274. doi: 10.1016/j.tins.2013.03.001

Deco, G., Jirsa, V. K., Robinson, P. A., Breakspear, M., and Friston, K. J. (2008). The dynamic brain: from spiking neurons to neural masses and cortical fields. PLoS Comput. Biol. 4:e1000092. doi: 10.1371/journal.pcbi.1000092

Dehaene, S., and Cohen, L. (2007). Cultural recycling of cortical maps. Neuron 56, 384-398. doi: 10.1016/j.neuron.2007.10.004

Dehaene, S., Meyniel, F., Wacongne, C., Wang, L., and Pallier, C. (2015). The neural representation of sequences: from transition probabilities to algebraic patterns and linguistic trees. Neuron 88, 2-19. doi: 10.1016/j.neuron.2015. 09.019

Deneve, S. (2008). Bayesian spiking neurons I: inference. Neural Comput. 20, 91-117. doi: 10.1162/neco.2008.20.1.91

Di Bono, M. G., and Zorzi, M. (2013). Deep generative learning of locationinvariant visual word recognition. Front. Psychol. 4:635. doi: 10.3389/fpsyg. 2013.00635

Elman, J. L., Bates, E., Johnson, M., Karmiloff-smith, A., Parisi, D., and Plunkett, K. (1996). Rethinking Innateness: A Connectionist Perspective on Development. Cambridge, MA: MIT Press.

Engel, A. K., Fries, P., and Singer, W. (2001). Dynamic predictions: oscillations and synchrony in top-down processing. Nat. Rev. Neurosci. 2, 704-716. doi: 10. $1038 / 35094565$

Farah, M. J. (2004). Visual Agnosia. Cambridge, MA: MIT Press.

Feigin, V. L., Forouzanfar, M. H., Krishnamurthi, R., Mensah, G. A., Connor, M., Bennett, D. A., et al. (2014). Global and regional burden of stroke during 1990-2010: findings from the Global Burden of Disease Study 2010. Lancet 383, 245-255. doi: 10.1016/s0140-6736(13)61953-4

Felleman, D. J., and Van Essen, D. C. (1991). Distributed hierarchical processing in the primate cerebral cortex. Cereb. Cortex 1, 1-47. doi: 10.1093/ cercor/1.1.1

Fiser, J., Berkes, P., Orbán, G., and Lengyel, M. (2010). Statistically optimal perception and learning: from behavior to neural representations. Trends Cogn. Sci. 14, 119-130. doi: 10.1016/j.tics.2010.01.003

Friston, K. J. (2010). The free-energy principle: a unified brain theory? Nat. Rev. Neurosci. 11, 127-138. doi: 10.1038/nrn2787

Gao, J., Barzel, B., and Barabási, A.-L. (2016). Universal resilience patterns in complex networks. Nature 530, 307-312. doi: 10.1038/nature16948

Gerstner, W., Sprekeler, H., and Deco, G. (2012). Theory and simulation in neuroscience. Science 338, 60-65. doi: 10.1126/science.1227356

Ghahramani, Z. (2015). Probabilistic machine learning and artificial intelligence. Nature 521, 452-459. doi: 10.1038/nature14541

Gilbert, C. D., and Sigman, M. (2007). Brain states: top-down influences in sensory processing. Neuron 54, 677-696. doi: 10.1016/j.neuron.2007.05.019 
Gläscher, J., Daw, N. D., Dayan, P., and O’Doherty, J. P. (2010). States versus rewards: dissociable neural prediction error signals underlying model-based and model-free reinforcement learning. Neuron 66, 585-595. doi: 10.1016/j. neuron.2010.04.016

Greicius, M. D., Krasnow, B., Reiss, A. L., and Menon, V. (2003). Functional connectivity in the resting brain: a network analysis of the default mode hypothesis. Proc. Natl. Acad. Sci. U S A 100, 253-258. doi: 10.1073/pnas. 0135058100

Griffiths, T. L., Kemp, C., and Tenenbaum, J. B. (2008). "Bayesian models of cognition," in Cambridge Handbook of Computational Cognitive Modeling, ed. R. Sun (Cambridge, MA: Cambridge University Press), 59-100.

Güçlü, U., and van Gerven, M. A. J. (2015). Deep neural networks reveal a gradient in the complexity of neural representations across the ventral stream. J. Neurosci. 35, 10005-10014. doi: 10.1523/JNEUROSCI.5023-14.2015

Hinton, G. E. (2002). Training products of experts by minimizing contrastive divergence. Neural Comput. 14, 1771-1800. doi: 10.1162/089976602760 128018

Hinton, G. E. (2007). Learning multiple layers of representation. Trends Cogn. Sci. 11, 428-434. doi: 10.1016/j.tics.2007.09.004

Hinton, G. E., Dayan, P., Frey, B., and Neal, R. M. (1995). The "wake-sleep" algorithm for unsupervised neural networks. Science 268, 1158-1161.

Hinton, G. E., and Salakhutdinov, R. (2006). Reducing the dimensionality of data with neural networks. Science 313, 504-507. doi: 10.1126/science.1127647

Hinton, G. E., and Shallice, T. (1991). Lesioning an attractor network: investigations of acquired dyslexia. Psychol. Rev. 98, 74-95. doi: 10.1037/0033295x.98.1.74

Humphreys, G. W., and Forde, E. M. (2001). Hierarchies, similarity and interactivity in object recognition: "category-specific" neuropsychological deficits. Behav. Brain Sci. 24, 453-476; discussion 476-509.

Itti, L., and Baldi, P. (2009). Bayesian surprise attracts human attention. Vision Res. 49, 1295-1306. doi: 10.1016/j.visres.2008.09.007

Jirsa, V. K., Sporns, O., Breakspear, M., Deco, G., and McIntosh, A. R. (2010). Towards the virtual brain: network modeling of the intact and the damaged brain. Arch. Ital. Biol. 148, 189-205.

Joanisse, M., and Seidenberg, M. S. (1999). Impairments in verb morphology after brain injury: a connectionist model. Proc. Natl. Acad. Sci. U S A 96, 7592-7597. doi: $10.1073 /$ pnas.96.13.7592

Jordan, M. I., and Sejnowski, T. J. (2001). Graphical Models: Foundations of Neural Computation. Cambridge, MA: MIT Press.

Kelso, J. A. S. (2012). Multistability and metastability: understanding dynamic coordination in the brain. Philos. Trans. R. Soc. Lond. B Biol. Sci. 367, 906-918. doi: 10.1098/rstb.2011.0351

Khaligh-Razavi, S. M., and Kriegeskorte, N. (2014). Deep supervised, but not unsupervised, models may explain IT cortical representation. PLoS Comput. Biol. 10:e1003915. doi: 10.1371/journal.pcbi.1003915

Kirkpatrick, S., Gelatt, C. Jr., and Vecchi, M. (1983). Optimization by simmulated annealing. Science 220, 671-680. doi: 10.1126/science.220.4598.671

Koller, D., and Friedman, N. (2009). Probabilistic Graphical Models: Principles and Techniques. Cambridge, MA: The MIT Press.

Lake, B. M., Salakhutdinov, R., and Tenenbaum, J. B. (2015). Humal-level concept learning through probabilistic program induction. Science 350, 1332-1338. doi: 10.1126/science.aab3050

Le, Q. V., Ranzato, M. A., Monga, R., Devin, M., Chen, K., Corrado, G. S., et al. (2012). "Building high-level features using large scale unsupervised learning," in Proceedings of the 29th International Conference on Machine Learning (Edinburgh, Scotland, UK).

LeCun, Y., Bengio, Y., and Hinton, G. E. (2015). Deep learning. Nature 521, 436-444. doi: 10.1038/nature14539

Lee, T. S., and Mumford, D. (2003). Hierarchical bayesian inference in the visual cortex. J. Opt. Soc. Am. A Opt. Image Sci. Vis. 20, 1434-1448. doi: 10.1364/josaa. 20.001434

Ma, W. J., Beck, J. M., Latham, P. E., and Pouget, A. (2006). Bayesian inference with probabilistic population codes. Nat. Neurosci. 9, 1432-1438. doi: 10. 1038/nn 1790

Manford, M., and Andermann, F. (1998). Complex visual hallucinations. Clinical and neurobiological insights. Brain 121, 1819-1840. doi: 10.1093/brain/121.10. 1819

Mathers, C., Fat, D. M., and Boerma, T. J. (2008). The Global Burden of Disease: 2004 Update. Geneva, Switzerland: World Health Organization.
Mavritsaki, E., Heinke, D., Allen, H., Deco, G., and Humphreys, G. W. (2011). Bridging the gap between physiology and behavior: evidence from the sSoTS model of human visual attention. Psychol. Rev. 118, 3-41. doi: 10. 1037/a0021868

McClelland, J. L. (2013). Integrating probabilistic models of perception and interactive neural networks: a historical and tutorial review. Front. Psychol. 4:503. doi: 10.3389/fpsyg.2013.00503

McClelland, J. L., McNaughton, B., and O'Reilly, R. C. (1995). Why there are complementary learning systems in the hippocampus and neocortex: insights from the successes and failures of connectionist models of learning and memory. Psychol. Rev. 102, 419-457. doi: 10.1037/0033-295x.102. 3.419

Medaglia, J. D., Lynall, M.-E., and Bassett, D. S. (2015). Cognitive network neuroscience. J. Cogn. Neurosci. 27, 1471-1491. doi: 10.1162/jocn_a_ 00810

Mnih, V., Kavukcuoglu, K., Silver, D., Rusu, A. A., Veness, J., Bellemare, M. G., et al. (2015). Human-level control through deep reinforcement learning. Nature 518, 529-533. doi: 10.1038/nature14236

Nessler, B., Pfeiffer, M., Buesing, L., and Maass, W. (2013). Bayesian computation emerges in generic cortical microcircuits through spike-timingdependent plasticity. PLoS Comput. Biol. 9:e1003037. doi: 10.1371/journal.pcbi. 1003037

Newman, M. (2010). Networks: An Introduction. New York, NY: Oxford University Press.

Ngiam, J., Khosla, A., Kim, M., Nam, J., Lee, H., and Ng, A. Y. (2011). “Multimodal deep learning," in Proceedings of the 28th International Conference on Machine Learning (ICML-11), Bellevue, WA, 689-696.

O'Reilly, R. C., and Munakata, Y. (2000). Computational Exploration in Cognitive Neuroscience. Cambridge, MA: MIT Press.

Park, H.-J., and Friston, K. J. (2013). Structural and functional brain networks: from connections to cognition. Science 342:1238411. doi: 10.1126/science. 1238411

Pecevski, D., Buesing, L., and Maass, W. (2011). Probabilistic inference in general graphical models through sampling in stochastic networks of spiking neurons. PLoS Comput. Biol. 7:e1002294. doi: 10.1371/journal.pcbi. 1002294

Plaut, D. C., and Behrmann, M. (2011). Complementary neural representations for faces and words: a computational exploration. Cogn. Neuropsychol. 28, 251-275. doi: 10.1080/02643294.2011.609812

Plaut, D. C., and Shallice, T. (1993). Deep dyslexia: a case study of connectionist neuropsychology. Cogn. Neuropsychol. 10, 377-500. doi: 10. $1080 / 02643299308253469$

Pouget, A., and Driver, J. (2000). Relating unilateral neglect to the neural coding of space. Curr. Opin. Neurobiol. 10, 242-249. doi: 10.1016/s0959-4388(00) 00077-5

Raichle, M. E. (2015). The restless brain: how intrinsic activity organizes brain function. Philos. Trans. R. Soc. Lond. B Biol. Sci. 370:20140172. doi: 10.1098/ rstb.2014.0172

Raina, R., Madhavan, A., and Ng, A. Y. (2009). "Large-scale deep unsupervised learning using graphics processors," in International Conference on Machine Learning, (New York, NY: ACM Press), 873-880.

Rao, R. P. N. (2004). Bayesian computation in recurrent neural circuits. Neural Comput. 16, 1-38. doi: 10.1162/08997660460733976

Reichert, D. P., Series, P., and Storkey, A. (2013). Charles bonnet syndrome: evidence for a generative model in the cortex? PLoS Comput. Biol. 9:e1003134. doi: 10.1371/journal.pcbi.1003134

Roitman, J. D., Brannon, E. M., and Platt, M. L. (2007). Monotonic coding of numerosity in macaque lateral intraparietal area. PLoS Biol. 5:e208. doi: 10. 1371/journal.pbio.0050208

Rotzer, S., Kucian, K., Martin, E., von Aster, M., Klaver, P., and Loenneker, T. (2008). Optimized voxel-based morphometry in children with developmental dyscalculia. Neuroimage 39, 417-422. doi: 10.1016/j.neuroimage.2007. 08.045

Rumelhart, D. E., and McClelland, J. L. (1986). Parallel Distributed Processing: Explorations in the Microstructure of Cognition, Vol. 1: Foundations. Cambridge, MA: MIT Press.

Sillito, A. M., Cudeiro, J., and Jones, H. E. (2006). Always returning: feedback and sensory processing in visual cortex and thalamus. Trends Neurosci. 29, 307-316. doi: 10.1016/j.tins.2006.05.001 
Silver, D., Huang, A., Maddison, C. J., Guez, A., Sifre, L., van den Driessche, G., et al. (2016). Mastering the game of Go with deep neural networks and tree search. Nature 529, 484-489. doi: 10.1038/nature16961

Snodgrass, J. G., and Vanderwart, M. (1980). A standardized set of 260 pictures: norms for name agreement, image agreement, familiarity and visual complexity. J. Exp. Psychol. Hum. Learn. 6, 174-215. doi: 10.1037/0278-7393. 6.2.174

Stoianov, I., and Zorzi, M. (2012). Emergence of a "visual number sense" in hierarchical generative models. Nat. Neurosci. 15, 194-196. doi: 10.1038/nn. 2996

Stoianov, I., and Zorzi, M. (2013). "Developmental trajectories of numerosity perception," in Poster Presented at the Workshop: Interactions Between Space, Time and Number: 20 Years of Research (Paris).

Stoianov, I., Zorzi, M., and Umiltà, C. (2004). The role of semantic and symbolic representations in arithmetic processing: insights from simulated dyscalculia in a connectionist model. Cortex 40, 194-196. doi: 10.1016/s0010-9452(08) 70948-1

Sutskever, I., Hinton, G. E., and Taylor, G. (2008). The recurrent temporal restricted Boltzmann machine. Adv. Neural Inf. Process. Syst. 20, 1601-1608.

Tenenbaum, J. B., Kemp, C., Griffiths, T. L., and Goodman, N. D. (2011). How to grow a mind: statistics, structure and abstraction. Science 331, 1279-1285. doi: 10.1126/science.1192788

Testolin, A., Stoianov, I., De Filippo De Grazia, M., and Zorzi, M. (2013). Deep unsupervised learning on a desktop PC: a primer for cognitive scientists. Front. Psychol. 4:251. doi: 10.3389/fpsyg.2013.00251
Testolin, A., Stoianov, I., Sperduti, A., and Zorzi, M. (2016). Learning orthographic structure with sequential generative neural networks. Cogn. Sci. 40, 579-606. doi: $10.1111 / \operatorname{cogs} .12258$

Testolin, A., Stoianov, I., and Zorzi, M. (under review). Human-like letter perception emerges from unsupervised deep learning and recycling of natural image statistics.

Varela, F., Lachaux, J. P., Rodriguez, E., and Martinerie, J. (2001). The brainweb: phase synchronization and large-scale integration. Nat. Rev. Neurosci. 2, 229-239. doi: 10.1038/35067550

Zorzi, M., Stoianov, I., and Umiltà, C. (2005). "Computational modeling of numerical cognition," in Handbook of Mathematical Cognition, ed. J. Campbell (New York, NY: Psychology Press), 67-84.

Zorzi, M., Testolin, A., and Stoianov, I. (2013). Modeling language and cognition with deep unsupervised learning: a tutorial overview. Front. Psychol. 4:515. doi: 10.3389/fpsyg.2013.00515

Conflict of Interest Statement: The authors declare that the research was conducted in the absence of any commercial or financial relationships that could be construed as a potential conflict of interest.

Copyright (C) 2016 Testolin and Zorzi. This is an open-access article distributed under the terms of the Creative Commons Attribution License (CC BY). The use, distribution and reproduction in other forums is permitted, provided the original author(s) or licensor are credited and that the original publication in this journal is cited, in accordance with accepted academic practice. No use, distribution or reproduction is permitted which does not comply with these terms. 